\title{
Optical signatures of semiconducting transition metal dichalcogenide nanosheets
}

Weijie Zhao ${ }^{a, b}$, Ricardo Mendes Ribeiro ${ }^{c}$, Goki Eda ${ }^{a, b, e *}$

${ }^{a}$ Department of Physics, National University of Singapore, 2 Science Drive 3, Singapore 117542

${ }^{b}$ Graphene Research Centre, National University of Singapore, 6 Science Drive 2, Singapore 117546

${ }^{c}$ Center of Physics and Department of Physics, University of Minho, PT-4710-057, Braga, Portugal

e Department of Chemistry, National University of Singapore, 3 Science Drive 3, Singapore 117543

KEYWORDS: Two-dimensional materials, transition metal dichalcogenide, molybdenum disulfide, photoluminescence, phase transformation, electronic structure 


\section{Conspectus}

Two-dimensional (2D) crystals derived from transition metal dichalcogenides (TMDs) are intriguing materials that offer a unique platform to study fundamental many-body physical phenomena as well as to build novel functional materials and devices. Semiconducting group 6 TMDs such as $\mathrm{MoS}_{2}$ and $\mathrm{WSe}_{2}$ are known for their large optical absorption coefficient and their potential for high efficiency photovoltaics and photodetectors. Monolayer sheets of these compounds are flexible, stretchable, and soft semiconductors with a direct band gap in contrast to their well-known bulk crystals that are rigid and hard indirect gap semiconductors. Recent intense research has been motivated by the distinct electrical, optical, and mechanical properties of these TMD crystals in the ultimate thickness regime.

As a semiconductor with a band gap in the visible to near-IR frequencies, these $2 \mathrm{D} \mathrm{MX}_{2}$ materials $(\mathrm{M}=\mathrm{Mo}, \mathrm{W} ; \mathrm{X}=\mathrm{S}, \mathrm{Se})$ exhibit distinct excitonic absorption and emission features. In this Account, we discuss how optical spectroscopy of these materials allows investigation of their electronic properties and the relaxation dynamics of excitons. We first discuss the basic electronic structure of 2D TMDs highlighting the key features of the dispersion relation. With the help of theoretical calculations, we further discuss how photoluminescence energy of direct and indirect excitons provide a guide to understanding the evolution of the electronic structure as a function of the number of layers. We also highlight the behavior of the two competing conduction valleys and their role in the optical processes.

Intercalation of group 6 TMDs by alkali metals results in the structural phase transformation with corresponding semiconductor-to-metal transition. Monolayer TMDs obtained by intercalationassisted exfoliation retains the metastable metallic phase. Mild annealing, however, destabilizes 
the metastable phase and gradually restores the original semiconducting phase. Interestingly, the semiconducting $2 \mathrm{H}$ phase, metallic $1 \mathrm{~T}$ phase, and a charge-density-wave-like $1 \mathrm{~T}$ ' phase can coexist within a single crystalline monolayer sheet. We further discuss the electronic properties of the restacked films of chemically exfoliated $\mathrm{MoS}_{2}$.

Finally, we focus on the strong optical absorption and related exciton relaxation in monolayer and bilayer $\mathrm{MX}_{2}$. Monolayer $\mathrm{MX}_{2}$ absorbs as much as $30 \%$ of incident photons in the blue portion of the visible light despite being atomically thin. This giant absorption is attributed to nesting of the conduction and valence bands, which leads to diversion of optical conductivity. We describe how the relaxation pathway of excitons depends strongly on the excitation energy. Excitation at the band nesting region is of unique significance as it leads to relaxation of electrons and holes with opposite momentum and spontaneous formation of indirect excitons. 
Since single-layer graphene was isolated by a piece of adhesive tape and its extraordinary properties were revealed by a series of experimental studies ${ }^{1-3}$, graphene research has grown at a tremendous speed and branched into numerous multidisciplinary areas with activities at both fundamental and technologically relevant levels. Reminiscent of this explosion of interest, the past few years have seen a surge of intense research on two-dimensional (2D) materials based on transition metal dichalcogenides (TMDs). ${ }^{4-7}$ The large family of TMDs sharing a chemical formula of $\mathrm{MX}_{2}(\mathrm{M}$ : transition metal, X: chalcogen) and van der Waals layer structure have been studied in their bulk forms for over half a century ${ }^{8}$. Molybdenum disulfide $\left(\mathrm{MoS}_{2}\right)$ is an archetypal TMD known for its use as solid-state lubricant and catalyst. The ultrathin " $2 \mathrm{D}$ " crystals of $\mathrm{MoS}_{2}$ dates back to the initial work by Frindt and coworkers in the 60 's ${ }^{9}$. The recent interest in 2D TMDs was triggered by the observation of direct gap photoluminescence (PL) from monolayer $\mathrm{MoS}_{2}$ by two independent research teams ${ }^{10,11}$. They found that the material, which is known to be an indirect gap semiconductor in the bulk form, curiously became a direct gap semiconductor when thinned to a monolayer. It was further shown that other group 6 TMDs such as $\mathrm{MoSe}_{2}, \mathrm{WS}_{2}$ and $\mathrm{WSe}_{2}$ show a similar behavior and become direct gap semiconductors when their monolayer is isolated ${ }^{12-14}$. Due to their mechanical flexibility, large carrier mobility, unique symmetry, and gate-tunability, 2D semiconductors of this family are attractive fundamental building blocks for novel optoelectronics and photonics applications ${ }^{4-7}$.

Luminescence of the 2D layers of group 6 TMDs occurs in the visible to near-IR frequencies and reveals a great deal of information about their electronic structure, many-body interaction, excited-state coherence, doping, defects, and $\operatorname{strain}^{10,} 11,15-23$. Optical spectroscopy has significantly facilitated the understanding these effects and their interplay. In this Account, we discuss our recent efforts on investigating the electronic properties of 2D TMDs through optical 
spectroscopy. This article is not meant to be a comprehensive review, which are available elsewhere ${ }^{4-7}$, but is aimed at highlighting some unique features of group 6 2D TMDs such as their phase change properties, layer-dependent evolution of electronic structures, and giant optical absorption due to band nesting.

\section{Electronic structure}

Many interesting properties of 2D materials arise from the unique dispersion of the electronic states that extend over the 2D plane. Precise description of their electronic structure typically requires rigorous density functional theory (DFT) calculations. The basic electronic properties of TMDs can be, however, predicted from the ligand field splitting of the non-bonding transition metal d-orbitals and the filling of these orbitals. ${ }^{4}$ All layered TMDs crystallize in either octahedral or trigonal prismatic coordination and the group $6 \mathrm{MX}_{2}$ exhibits the latter in its most stable form (typically referred to as $2 \mathrm{H}$ phase). In the $2 \mathrm{H}$ phase, the ligand field splitting of the $\mathrm{d}$ orbitals leads to the formation of $\mathrm{d}_{z}^{2}\left(\mathrm{a}_{1}{ }^{\prime}\right), \mathrm{d}_{\mathrm{x}-\mathrm{y}, \mathrm{xy}}^{2}{ }^{2}\left(\mathrm{e}^{\prime}\right)$, and $\mathrm{d}_{\mathrm{xz}, \mathrm{yz}}\left(\mathrm{e}^{\prime \prime}\right)$ bands separated by an energy gap. ${ }^{4}$ The two $d$ electrons of the transition metals fill up the $a_{1}{ }^{\prime}$ orbital, making it a semiconductor (Figure 1a). Octahedrally coordinated TMDs (often referred to as 1T phase) form degenerate $d_{x y, y z, z x}\left(t_{2 g}\right)$ and $d_{z}{ }^{2}{ }^{2}-y{ }^{2}\left(e_{g}\right)$ orbitals. Generally, group 6 TMDs are not stable in the octahedral coordination but intercalation by alkali metals ${ }^{4,24}$ and high dose electron irradiation ${ }^{25}$ are known to induce formation of this phase. Because the lower lying $\mathrm{t}_{2 \mathrm{~g}}$ orbital is only partially filled, group 6 TMDs in the 1T phase are metallic in electronic character (Figure 1b). The M-X bonding states $(\sigma)$ appear deep in the valence band indicating its strong covalent character. The precise position of the $\mathrm{M}-\mathrm{X}$ antibonding states $\left(\sigma^{*}\right)$ has been debated ${ }^{26}$ but for clarity it is shown to be above the non-bonding states in Figure 1a and $\mathrm{b}$. 
Further understanding of the electronic structure requires consideration of the energy dispersion. Figure 1c shows the DFT calculation of the band structure of monolayer $2 \mathrm{H}-\mathrm{MoS}_{2}$. The key features of the band structure are the two valence band hills and the two conduction band valleys. The valence band hills are at the center $(\Gamma)$ and corners $(\mathrm{K})$ of the Brillouin zone. The conduction band valleys are at the $\mathrm{K}$ point and the $\Lambda$ point, which is midway between the $\mathrm{K}-\Gamma$ line. Note that the valence band at the $\mathrm{K}$ point is split due to spin-orbit coupling. The DFT calculation shows that monolayer $\mathrm{MoS}_{2}$ is a direct gap semiconductor with the conduction band minimum (CBM) and the valence band maximum (VBM) coinciding at the $\mathrm{K}$ point. In contrast, bilayer $\mathrm{MoS}_{2}$ is an indirect gap semiconductor with $\mathrm{CBM}$ at the $\mathrm{K}$ point and the VBM at the $\Gamma$ point (Not shown). While there are slight variations in the DFT results reported by different groups, all calculations agree that the band gap is direct for monolayer $\mathrm{MoS}_{2}$.

The effect of the number of layers appears most strongly at the conduction band valley at the $\Lambda$ point $\left(\Lambda_{\mathrm{c}}\right)$ and the valence band hill at the $\Gamma$ point $\left(\Gamma_{\mathrm{v}}\right)$. Careful examination reveals that the wavefunction associated with these points consists of an admixture of the metal d orbitals and the chalcogen $p_{z}$ orbitals. In contrast, the wavefunctions at the $K_{c}$ and $K_{v}$ regions have predominantly $d$ character. The charge density of the $p_{z}$ orbitals extends at the outer surface of the layer while the $\mathrm{d}$ electrons are confined in the middle of the X-M-X sandwich (Figure 1d) ${ }^{11}$. Thus, the evolution of the band structure with increasing number of layers results primarily due to the interaction of the chalcogen $\mathrm{p}_{z}$ orbitals with those of the neighboring layers.

\section{Photoluminescence}

Splendiani et al. ${ }^{11}$ and Mak et al. ${ }^{10}$ systematically studied the evolution of the optical properties of mechanically exfoliated $\mathrm{MoS}_{2}$ flakes as a function of the number of layers. The authors 
observed bright PL from monolayer $\mathrm{MoS}_{2}$ and progressively weaker emission from bilayer and thicker multiplayer samples (Figure 2a). This observation is contrary to the expectation that the optical response scales with the volume of the material. The bright PL from monolayer $\mathrm{MoS}_{2}$ can be, however, explained by the indirect-to-direct band gap cross over at the monolayer limit. As an indirect gap semiconductor, the emission quantum yield of bulk $\mathrm{MoS}_{2}$ is negligible because the radiative recombination of indirect excitons is a phonon-assisted second order process and is slower than non-radiative decay rates. On the other hand, monolayer $\mathrm{MoS}_{2}$ is a direct gap semiconductor and the radiative recombination of excitons is substantially more efficient. The bright emission centered at $1.9 \mathrm{eV}$ from monolayer $\mathrm{MoS}_{2}$ is therefore associated with the band gap at the K point of the Brillouin zone.

Multilayer $\mathrm{MoS}_{2}$ flakes exhibit weak PL with multiple peaks. The lowest energy peak is due to indirect excitons and the higher energy peaks at $\sim 1.9$ and $2.05 \mathrm{eV}$ are attributed to hot electron direct gap emission at the $\mathrm{K}$ point. The shift of indirect and direct emission energies as a function of the number of layers reveals the evolution of the electronic structure (Figure 2b). Specifically, the indirect gap energy increases rapidly with decreasing number of layers while the direct gap energy remains largely unaffected. The indirect-to-direct gap crossover occurs when the indirect gap energy exceeds the direct gap energy at the monolayer limit.

Our research group ${ }^{12}$ and others ${ }^{13,14}$ have shown that the trend of indirect-to-direct gap crossover is not a unique phenomenon to $\mathrm{MoS}_{2}$ but is also seen in other group 6 TMDs such as $\mathrm{MoSe}_{2}$, $\mathrm{WS}_{2}$, and $\mathrm{WSe}_{2}$, which are isoelectronic to $\mathrm{MoS}_{2}$. The basic trends are the same: they become a direct gap semiconductor and exhibit bright band gap PL only when they are thinned to a monolayer (Figure 2c). The quantum yield of emission for bilayer samples is typically 10 to 100 times smaller than that of monolayer samples. The optical gap of monolayers determined from 
PL spectroscopy is $\sim 1.83, \sim 1.58, \sim 1.98$ and $\sim 1.63 \mathrm{eV}$ for $\mathrm{MoS}_{2}, \mathrm{MoSe}_{2}, \mathrm{WS}_{2}$, and $\mathrm{WSe}_{2}$, respectively (Figure 2e).

\section{Absorption}

In the near-IR to ultraviolet frequencies, group 6 TMDs exhibit characteristic absorption peaks that can be attributed to excitonic and interband resonances ${ }^{8,10,11}$. The exciton features are typically dominant and obscure the onset of free carrier absorption. In fact, this has been the key issue for accurately determining the exciton binding energy ${ }^{27}$. The absorption features for bulk crystals and their origins have been discussed in several reports including the detailed low temperature studies by Beal et $\mathrm{al}^{28}$ and Bromley et al. ${ }^{29}$. The $\mathrm{A}$ and $\mathrm{B}$ exciton resonances arise from optical excitation of electrons from the spin-orbit-split valance band hill to the degenerate conduction band valley at the $\mathrm{K}$ point. The $\mathrm{C}$ absorption peak, which appears $(2.6 \sim 2.8 \mathrm{eV})$ at higher energies, has been attributed to band nesting, which leads to a singularity in the joint density of states (JDOS $)^{30}$. The unique consequence of band nesting will be discussed in the later section. The $\mathrm{A}^{\prime}$ and $\mathrm{B}^{\prime}$ peaks observed in selenides and tellurides have been attributed to splitting of the A and B exciton states but their origin remains unclear. Monolayer crystals typically absorb $\sim 10 \%$ of incident photons at its band gap A exciton resonance and more strongly $(\sim 30 \%)$ at the $\mathrm{C}$ peak resonance. In comparison to the optical absorption of graphene, which is about $2.3 \%{ }^{31}$, these large values highlight the unusual light-matter interaction in these monolayer crystals ${ }^{32}$.

Similar to PL, the shift of the absorption resonances as a function of the number of layers reflects the evolution of the band structure. The blue shift in the absorption peaks with reducing crystal thickness in $\mathrm{MoS}_{2}$ and $\mathrm{WSe}_{2}$ was initially studied by Frindt and coworkers in the 1960's ${ }^{9}$. The 
authors curiously highlighted the deviation in the scaling behavior of the optical gap with the crystal thickness when the $\mathrm{WSe}_{2}$ samples were thinner than $4 \mathrm{~nm}$. The origin of this deviation remained elusive until recently when the accurate picture of the band structure became available.

\section{$\mathrm{WS}_{2}$ and $\mathrm{WSe}_{2}$}

In order to gain insight into the origin of the optical transitions, we investigated the confinementinduced shift of exciton absorption and emission peaks in 1 to 5 layer (L) $\mathrm{WS}_{2}$ and $\mathrm{WSe}_{2}$. Note that the layer number can be confirmed readily by atomic force microscopy and Raman spectroscopy $^{13,33,34}$. Monolayer of both materials exhibits bright direct gap emission while multilayers emit weakly at two distinct energies corresponding to direct and indirect transitions (Figure 3a). We also observed weak emission peaks at higher energies due to hot exciton recombination. The shift of emission and absorption peaks summarized in Figure $3 \mathrm{~b}$ clearly indicates that the scaling factor is unique to each exciton resonance. The A and B exciton energy is only weakly dependent on the crystal thickness while A', B' and C peak energies increase more rapidly with reduction in the number of layers. The A' and B' excitons in $\mathrm{WSe}_{2}$, which had been attributed to splitting of the A and B exciton energy levels, show similar trends as the indirect exciton peak. This observation suggests that these optical transitions are more strongly associated with the $\mathrm{p}_{\mathrm{z}}$ orbitals of the Se atoms rather than the metal d orbitals.

The confinement-induced change in the band gap of 2D semiconductors $\left(\Delta E_{\mathrm{g}}\right)$ is predicted to scale as $\Delta E_{\mathrm{g}}=\pi^{2} h^{2} / 8 \mu L_{\mathrm{z}}^{2}$ where $h$ is the Planck's constant, $\mu$ is the reduced mass of the exciton, and $L_{\mathrm{z}}$ is the thickness of the crystals ${ }^{35}$. This simple relation does not apply to the optical gap of TMD crystals in the few layer thickness regime for various reasons. First, the band structure of the material is dramatically modified with the relative shift of the conduction band valleys and 
valence band hills. Second, the dielectric properties are correspondingly altered, leading to the remarkable increase in the exciton binding energies with decreasing thickness ${ }^{36}$. Third, extrinsic effects such as unintentional doping by surface adsorbates ${ }^{37}$ and substrate ${ }^{38}$ become increasingly important with decreasing thickness and obscure the band gap absorption and emission.

\section{Conduction band minimum}

As discussed above, the band structures obtained from DFT calculations provide a useful guide for identifying the origin of the experimentally observed optical absorption and emission. However, identifying the origin of indirect band gap emission in few-layer $\mathrm{MX}_{2}$ has been a nontrivial task. This is because DFT results show little consensus on the conduction band structure of few-layer $\mathrm{MX}_{2}$. For bilayer $\mathrm{MoS}_{2}$ and $\mathrm{WS}_{2}$, some calculations $\mathrm{s}^{23,36,38-40}$ show that the CBM is located at the $\mathrm{K}$ point while others ${ }^{11,39-41}$ show that it is at the $\Lambda$ point. On the other hand, the DFT calculations consistently show the VBM to be at the $\Gamma$ point in few-layer $\mathrm{MX}_{2}$. This finding has been confirmed to agree with the experimental observations by angle-resolved photoemission spectroscopy (ARPES) ${ }^{42,43}$.

One important question is: where is the $\mathrm{CBM}$ in few-layer $\mathrm{MX}_{2}$, is it at the $\Lambda$ or $\mathrm{K}$ point? Despite the fundamental significance of the location of the CBM, direct investigation of the dispersion of unfilled conduction band states is generally a challenging task. In order to investigate the competition of the $\Lambda$ and $\mathrm{K}$ valley in few-layer $\mathrm{MX}_{2}$, we studied the temperature dependence of the radiative indirect recombination (see schematics in Figure $4 \mathrm{a}$ and b). ${ }^{44}$ Temperature is used as the knob to arbitrarily vary the lattice parameters through natural thermal expansion, which in turn leads to evolution of the band structure. 
We briefly explain how thermal expansion of the lattice modifies the band structure. The inplane expansion influences the overlap of the metal $\mathrm{d}$ orbitals, which are responsible for the $\mathrm{K}$ valleys and hills. Increase in the in-plane lattice constant by $\Delta a$ leads to reduction of the gap at the $\mathrm{K}$ point $\left(A=E\left(\mathrm{~K}_{\mathrm{c}}\right)-E\left(\mathrm{~K}_{\mathrm{v}}\right)\right)$ and corresponding slight reduction of the indirect gap between $\mathrm{K}_{\mathrm{c}}$ and $\Gamma_{\mathrm{v}}^{23,45}$. In contrast, the expansion of interlayer spacing primarily affects the $\Lambda_{\mathrm{c}}$ and $\Gamma_{\mathrm{v}}$ points because the $\mathrm{p}$ orbital energy of the chalcogen atoms are susceptible to changes in the interlayer coupling. According to the DFT calculations ${ }^{23,45}$, the $\Lambda$ valley and $\Gamma$ hill shift upwards and downwards, respectively, with increasing interlayer expansion, eventually leading to the band structure of monolayer $\mathrm{MX}_{2}$ at infinite interlayer spacing.

Thermal expansion the lattice leads to a composite effect of in-plane and out-of-plane expansion explained above. Using the experimentally measured thermal expansion coefficients for bulk $\mathrm{MoS}_{2}$ and $\mathrm{WSe}_{2},{ }^{46,47}$ we calculated the temperature dependence of the direct and indirect energy gaps. We found that the two indirect gap energies, $I_{1}=E\left(\mathrm{~K}_{\mathrm{c}}\right)-E\left(\Gamma_{\mathrm{v}}\right)$ and $I_{2}=E\left(\Lambda_{\mathrm{c}}\right)-\mathrm{E}\left(\Gamma_{\mathrm{v}}\right)$, exhibit opposite temperature dependence (Figure 4c). Thus, the temperature dependence of the indirect gap emission can help us identify whether the $\Lambda$ or $\mathrm{K}$ valley is involved in the transition. Further, by properly taking into account the exciton binding energies, the location of the CBM can be identified.

The temperature coefficient of the indirect emission energy for bilayer $\mathrm{WSe}_{2}$ was experimentally found to be negative (Figure 4d). This suggests that the indirect optical transition involves the K valley. In contrast, the temperature coefficient in $\mathrm{MoS}_{2}$ and $\mathrm{WS}_{2}$ is positive, suggesting that the $\Lambda$ valley is involved in the optical transition (Not shown in figure). Interestingly, thicker fewlayer $\mathrm{WSe}_{2}$ flakes exhibit two indirect emission peaks with opposite tempeature coefficients 
(Figure4d). These findings indicate that the conduction band valleys in $\mathrm{WSe}_{2}$ are nearly degenerate as indicated by the DFT results ${ }^{48}$.

\section{Phase transition}

Solution-based exfoliation of layered materials is a promising route to producing $2 \mathrm{D}$ crystals in large scale and implementing them into practical applications ${ }^{49-51}$. We recently showed that the exfoliated nanosheets of monolayer $\mathrm{MoS}_{2}$ can be used photosensitize a surface of $\mathrm{TiO}_{2}$ particle ${ }^{52}$. Intercalation-assisted, or chemical exfoliation is a technique that allows high yield preparation of monolayer TMDs in colloidal suspensions (Figure $5 \mathrm{a})^{53}$. The electronic quality of the derived nanosheets is, however, not clearly understood due to the structural changes that occur during the intercalation process.

It is well established that alkali metal intercalation of semiconducting $2 \mathrm{H}-\mathrm{MoS}_{2}$ results in its structural transformation to metallic $1 \mathrm{~T}-\mathrm{MoS}_{2}{ }^{24}$. This polymorphic phase transformation is one of the most intriguing attributes of TMDs. Earlier studies have shown that the $1 \mathrm{~T}$ phase is metastable and converts back to the stable $2 \mathrm{H}$ phase upon mild annealing ${ }^{54}$. However, the structure of the exfoliated material and the extent of the restoration of the original $2 \mathrm{H}$ phase have been debated ${ }^{55}$.

We investigated the structural evolution of chemically exfoliated $\mathrm{MoS}_{2}$ and $\mathrm{WS}_{2}$ through a series of spectroscopy and microscopy studies. X-ray photoelectron spectroscopy (XPS) analyses indicated that the $2 \mathrm{H}$ and $1 \mathrm{~T}$ phases coexist in the as-exfoliated materials at variable fractions ${ }^{54}$. From the area fraction of the peaks associated with the $2 \mathrm{H}$ and $1 \mathrm{~T}$ phase, we determined the phase composition changes as a function of the annealing temperature (Figure 5b). Our results show that the fraction of the $2 \mathrm{H}$ phase increases with annealing temperature, reaching 0.95 at 
$300{ }^{\circ} \mathrm{C}$. This observation is in agreement with the dramatic increase in the electrical resistivity of the material reflecting its transformation from the metallic $1 \mathrm{~T}$ phase to semiconducting $2 \mathrm{H}$ phase. Raman spectroscopy also indicates the gradual loss of $1 \mathrm{~T}$ phase and recovery of the $2 \mathrm{H}$ phase with annealing (Figure 5c).

The coexistence of the two phases prompts a new question: how are these phases distributed within the exfoliated material? Atomic resolution scanning transmission electron microscopy (STEM) of chemically exfoliated $\mathrm{MoS}_{2}$ and $\mathrm{WS}_{2}$ revealed that the $2 \mathrm{H}$ and $1 \mathrm{~T}$ phases exist in nanoscopic domains within individual sheets ${ }^{56}$. Figure $5 \mathrm{~d}$ shows the annular dark field (ADF) STEM images showing the different contrast variations corresponding to distinct phases. Due to the lattice matching, these domains form seamless, coherent interfaces. Interestingly, we also observed a charge-density-wave (CDW)-like phase consisting of a superlattice of Mo-Mo and $\mathrm{W}-\mathrm{W}$ zigzag chains ${ }^{56,57}$. This is a distorted $1 \mathrm{~T}$ phase which has been referred to as the $1 \mathrm{~T}^{\prime}$ or $\mathrm{ZT}$ phase $^{25,58}$. These STEM images provide a direct evidence of the CDW-like phase and resolve the earlier confusions ${ }^{56}$. The complex inhomogeneous structure, however, suggests that the synthesis protocols need to be improved in order to achieve an electronically ordered phase.

The optical absorption spectrum of as-produced chemically exfoliated $\mathrm{MoS}_{2}$ shows no clear characteristic peaks of pristine $2 \mathrm{H}-\mathrm{MoS}_{2}$ except for the excitonic features in the near-UV range $(200 \sim 300 \mathrm{~nm})$. The free-carrier-like broad absorption continuing towards the near-IR region below the optical gap of $2 \mathrm{H}-\mathrm{MoS}_{2}$ indicates that the electronic properties of the chemically exfoliated material are dominated by the metallic $1 \mathrm{~T}$ phase component. The progression of the $1 \mathrm{~T}$ to $2 \mathrm{H}-\mathrm{MoS}_{2}$ transition with annealing can be monitored by studying the emergence of the excitonic absorption from the $2 \mathrm{H}$ phase (Figure 5e). With increasing annealing temperature, the characteristic A, B and C exciton features of $2 \mathrm{H}-\mathrm{MoS}_{2}$ emerge and grow in intensity, consistent 
with the XPS and Raman results. Emergence of these features indicates restoration of $2 \mathrm{H}$ phase upon annealing.

Restoration of the $2 \mathrm{H}$ phase can be also verified by PL spectroscopy. As expected from the metallic character of $1 \mathrm{~T}-\mathrm{MoS}_{2}$, no luminescence is observed from the as-deposited monolayers. With progressive annealing, however, characteristic band gap PL features of monolayer $2 \mathrm{H}-$ $\mathrm{MoS}_{2}$ are observed. The emission spectrum is nearly identical to that of mechanically exfoliated monolayer samples, indicating that the electronic quality of the original material is largely recovered even after the harsh chemical exfoliation processes and substantial structural transitions.

It is also worth noting the evolution of the emission spectra of restacked multilayers. Figure $5 f$ shows the drop in the emission intensity and the gradual red-shift with increasing film thickness. Although indirect emission peak was not observed, these trends are similar to those observed in mechanically exfoliated materials, indicating strong interlayer coupling despite the stacking disorder. Thus, thickness control via solution-processing offers a versatile route to tuning the electronic structure.

\section{Band nesting and photocarrier relaxation}

The large absorption coefficient of semiconducting TMDs has long attracted interest in their use as the active layer in photovoltaic devices ${ }^{59}$. While the absorption in absolute terms is expected to be negligible in the $2 \mathrm{D}$ limit where the interaction length is only 3 -atom long, experiments have shown that a single layer $\mathrm{MX}_{2}$ absorbs as much as $30 \%$ of incident photons in the blue

portion of the visible spectrum ${ }^{60}$. According to DFT calculations ${ }^{30,61}$, this extraordinary absorption is attributed to the band nesting effect where parallel conduction and valence bands 
give rise to a singularity feature in the JDOS. The optical conductivity is thus strongly enhanced for energy in resonance with the nesting region of the band structure.

Band nesting has unique implications in the dynamics of photocarrier relaxation. For the resonant excitation condition, the landscape of the energy bands in the momentum space dictates that the excited electron-hole pairs spontaneously form indirect excitons with electrons and holes relaxing with opposite momentum. Figure 6a schematically illustrates this scenario. The electrons and holes relax towards the $\Lambda_{\mathrm{c}}$ and $\Gamma_{\mathrm{v}}$ point, respectively. In monolayer $\mathrm{MX}_{2}$, electrons and holes need to further undergo intervalley scattering to relax to the CBM and VBM at the $\mathrm{K}$ point, respectively. Due to the involvement of intervalley phonons, relaxation of the photocarriers to the band edge is expected to be slow and inefficient in monolayers. On the other hand, $\Lambda_{\mathrm{c}}$ and $\Gamma_{\mathrm{v}}$ points are the band extrema in some bilayer $\mathrm{MX}_{2}$ as discussed above and photocarrier relaxation to the band edge is expected to be significantly faster.

We investigated the photocarrier relaxation pathway in $\mathrm{MX}_{2}$ with PL excitation (PLE) spectroscopy. The PLE intensity map of monolayer $\mathrm{MoS}_{2}$ (Figure 6b) shows that the emission energy is independent on the excitation energy and is consistent with the optical gap. The emission intensity, however, decreases with increasing excitation energy. In fact, the relative quantum yield (QY) is lowest when the excitation is in resonance with the strong absorption peak at $\sim 2.8 \mathrm{eV}$, which is attributed to band nesting. This observation is consistent with the spontaneous formation of indirect excitons with low radiative recombination efficiency.

We further studied the band nesting effect in bilayer $\mathrm{MoS}_{2}$ in which the band gap is indirect with CBM at the $\Lambda_{\mathrm{c}}$ point and VBM at the $\Gamma_{\mathrm{v}}$ point based on our analysis discussed in the earlier section. Interestingly, the direct and indirect emission peaks respond differently to the excitation 
energy (Figure 6d). The indirect emission $(\sim 1.5 \mathrm{eV})$ intensity is maximized when the excitation is in resonance with the $\mathrm{C}$ absorption peak. On the other hand, the enhancement of the direct emission peak $(\sim 1.85 \mathrm{eV})$ at this excitation energy is minor. These behaviors are consistent with the photocarrier dynamics predicted from the band structure and Monte Carlo simulations ${ }^{60}$.

\section{Summary and Outlook}

Many of the interesting electronic phenomena observed in 2D TMDs arise from the unique energy dispersion and strong correlation between electrons and holes. Optical spectroscopy is an effective approach to probing the essential features of the band structure and many-body effects. In particular, the emerging PL from mono- to few-layer $\mathrm{MX}_{2}$ plays a crucial role in revealing the evolution of the band structure with increasing number of layers. These studies have revealed that the optical gap of these 2D materials does not follow a simple scaling law with thickness due to the relative shift of the valleys and dramatic changes in the dielectric properties ${ }^{36}$. Theoretical calculations have been successful in explaining the origin of the experimentally observed optical transitions and their shifts.

Polymorphism of TMDs is a unique characteristic that makes them interesting for non-volatile memory and switching devices. The metallic $1 \mathrm{~T}$ phase of $\mathrm{MoS}_{2}$ and $\mathrm{WS}_{2}$ has been known from earlier studies. However, their coexistence within a single crystalline monolayer flake along with the highly anisotropic 1T' (or ZT) phase demonstrates the unusual versatility of these 2D

materials. The heterogeneous phase structure with metallic electrical conductivity has been of interest in the electroscatalysis research ${ }^{57}$. Stabilization of a desired phase remains an important challenge to overcome in order to exploit the full potential of their phase change properties. 
There have been a rapidly growing number of reports on the fabrication of optoelectronic devices based on semiconducting TMDs $^{4-7}$. While several groups reported promising results, the mechanisms responsible for the observed device performance remain largely elusive. Understanding the transient processes that occur between optical excitation and detection of electrical signals is crucial for optimizing of the device designs and identifying the ideal window of operation. The excitation dependence of the relaxation dynamics of excitons is an interesting consequence of the 2D band structure of these materials and merits further investigation in their exploitation.

Heterostructures of TMDs are a fundamental building block for realizing tailored optoelectronic functionalities ${ }^{6}$. Hybridization of the electronic states across van der Waals interfaces is expected to occur in vertical heterostructures according to DFT calculations ${ }^{62,}{ }^{63}$. However, there is currently a gap between the experimental observations and theoretical predictions. The heterostructures are significantly more complex than the single-component systems and the accurate interpretation of spectroscopic features require special care. Nevertheless, the electronic structure and the dynamic behavior of excitons in TMD heterostructures will be the key issues to address in the new phase of the 2D materials research.

\section{AUTHOR INFORMATION}

Corresponding Author

*g.eda@nus.edu.sg 


\section{Biographies}

\section{Dr. Weijie Zhao}

Dr. Zhao received his Ph.D. in 2011 from the Institute of Semiconductors, Chinese Academy of Sciences, Beijing. He joined the National University of Singapore as Research Fellow in 2012. His research interest lies in the optical spectroscopy of two-dimensional materials.

\section{Dr. Ricardo Mendes Ribeiro}

Dr. Ribeiro received his $\mathrm{PhD}$ from the University of Minho. In 2013, he received the Habilitation. He is Assistant Professor at University of Minho since 1999 and currently Visiting Associate Professor at the Graphene Research Centre, National University of Singapore. His main research interests are on Theoretical Solid State Physics, specially, in two-dimensional materials, like graphene. Other interests include Density Functional Theory study of nanocrystalline silicon in an amorphous silicon matrix, mesoscopic modelling of the evolution of surfaces and thin films, and mesoscopic modelling of electronic processes in conjugated polymers.

\section{Dr. Goki Eda}

Dr. Eda received his M.S. from Worcester Polytechnic Institute and Ph.D. from Rutgers University. He worked at Imperial College London as a Newton International Fellow of the Royal Society of UK. Dr. Eda joined the National University of Singapore and Graphene Research Centre as Assistant Professor and NRF Research Fellow in 2011.

\section{Author Contributions}


The manuscript was written through contributions of all authors. All authors have given approval to the final version of the manuscript.

\section{Notes}

The authors declare no competing financial interest.

\section{ACKNOWLEDGMENT}

G.E. acknowledges Singapore National Research Foundation for funding the research under

NRF Research Fellowship (NRF-NRFF2011-02). G.E. also thanks the Graphene Research Centre at the National University of Singapore for funding support. R.M.R is thankful for the financial support by FEDER through the COMPETE Program, by the Portuguese Foundation

for Science and Technology (FCT) in the framework of the Strategic Project PESTC/FIS/UI607/2011 and grant nr. SFRH/BSAB/1249/2012 and by the EC under Graphene Flagship (contract no. CNECT-ICT-604391).

\section{REFERENCES}

1. Castro Neto, A. H.; Guinea, F.; Peres, N. M. R.; Novoselov, K. S.; Geim, A. K., The electronic properties of graphene. Rev Mod Phys 2009, 81 (1), 109-162.

2. Geim, A. K.; Novoselov, K. S., The rise of graphene. Nat Mater 2007, 6 (3), 183-191.

3. Novoselov, K. S.; Fal'ko, V. I.; Colombo, L.; Gellert, P. R.; Schwab, M. G.; Kim, K., A roadmap for graphene. Nature 2012, 490 (7419), 192-200.

4. $\quad$ Chhowalla, M.; Shin, H. S.; Eda, G.; Li, L.-J.; Loh, K. P.; Zhang, H., The chemistry of two-dimensional layered transition metal dichalcogenide nanosheets. Nature Chemistry 2013, 5 (4), 263-275.

5. Wang, Q. H.; Kalantar-Zadeh, K.; Kis, A.; Coleman, J. N.; Strano, M. S., Electronics and optoelectronics of two-dimensional transition metal dichalcogenides. Nat Nanotechnol 2012, 7 (11), 699-712.

6. Geim, A. K.; Grigorieva, I. V., Van der Waals heterostructures. Nature 2013, 499 (7459), 419-25.

7. $\quad$ Butler, S. Z.; Hollen, S. M.; Cao, L.; Cui, Y.; Gupta, J. A.; Gutierrez, H. R.; Heinz, T. F.; Hong, S. S.; Huang, J.; Ismach, A. F.; Johnston-Halperin, E.; Kuno, M.; Plashnitsa, V. V.; 
Robinson, R. D.; Ruoff, R. S.; Salahuddin, S.; Shan, J.; Shi, L.; Spencer, M. G.; Terrones, M.; Windl, W.; Goldberger, J. E., Progress, challenges, and opportunities in two-dimensional materials beyond graphene. Acs Nano 2013, 7 (4), 2898-926.

8. Wilson, J. A.; Yoffe, A. D., The transition metal dichalcogenides discussion and interpretation of the observed optical, electrical and structural properties. Adv Phys 1969, 18 (73), 193-335.

9. Consadori, F.; Frindt, R. F., Crystal Size Effects on the Exciton Absorption Spectrum of WSe2. Phys Rev B 1970, 2 (12), 4893-4896.

10. Mak, K. F.; Lee, C.; Hone, J.; Shan, J.; Heinz, T. F., Atomically Thin MoS2: A New Direct-Gap Semiconductor. Phys Rev Lett 2010, 105 (13), 136805.

11. Splendiani, A.; Sun, L.; Zhang, Y. B.; Li, T. S.; Kim, J.; Chim, C. Y.; Galli, G.; Wang, F., Emerging Photoluminescence in Monolayer MoS2. Nano Lett 2010, 10 (4), 1271-1275.

12. Zhao, W. J.; Ghorannevis, Z.; Chu, L. Q.; Toh, M. L.; Kloc, C.; Tan, P. H.; Eda, G., Evolution of Electronic Structure in Atomically Thin Sheets of WS2 and WSe2. Acs Nano 2013, 7 (1), 791-797.

13. Zeng, H.; Liu, G. B.; Dai, J.; Yan, Y.; Zhu, B.; He, R.; Xie, L.; Xu, S.; Chen, X.; Yao, W.; Cui, X., Optical signature of symmetry variations and spin-valley coupling in atomically thin tungsten dichalcogenides. Sci Rep 2012, doi:10.1038/srep01608.

14. Tonndorf, P.; Schmidt, R.; Böttger, P.; Zhang, X.; Börner, J.; Liebig, A.; Albrecht, M.; Kloc, C.; Gordan, O.; Zahn, D. R. T.; Vasconcellos, S. M. d.; Bratschitsch, R., Photoluminescence emission and Raman response of monolayer MoS2, MoSe2, and WSe2. Optics Express 2013, 21, 4908.

15. Mak, K. F.; He, K. L.; Shan, J.; Heinz, T. F., Control of valley polarization in monolayer MoS2 by optical helicity. Nat Nanotechnol 2012, 7 (8), 494-498.

16. Zeng, H. L.; Dai, J. F.; Yao, W.; Xiao, D.; Cui, X. D., Valley polarization in MoS2 monolayers by optical pumping. Nat Nanotechnol 2012, 7 (8), 490-493.

17. Mak, K. F.; He, K. L.; Lee, C.; Lee, G. H.; Hone, J.; Heinz, T. F.; Shan, J., Tightly bound trions in monolayer MoS2. Nat Mater 2013, 12 (3), 207-211.

18. Radisavljevic, B.; Kis, A., Mobility engineering and a metal-insulator transition in monolayer MoS2. Nat Mater 2013, 12 (9), 815-20.

19. Jones, A. M.; Yu, H. Y.; Ghimire, N.; Wu, S. F.; Aivazian, G.; Ross, J. S.; Zhao, B.; Yan, J. Q.; Mandrus, D.; Xiao, D.; Yao, W.; Xu, X. D., Optical Generation of Excitonic Valley Coherence in Monolayer WSe2. Nat Nanotechnol 2013, 8, 634-638.

20. Ye, J. T.; Zhang, Y. J.; Akashi, R.; Bahramy, M. S.; Arita, R.; Iwasa, Y., Superconducting dome in a gate-tuned band insulator. Science 2012, 338 (6111), 1193-6.

21. Van der Zande, A. M.; Huang, P. Y.; Chenet, D. A.; Berkelbach, T. C.; You, Y.; Lee, G. H.; Heinz, T. F.; Reichman, D. R.; Muller, D. A.; Hone, J. C., Grains and grain boundaries in highly crystalline monolayer molybdenum disulphide. Nat Mater 2013, 12 (6), 554-61.

22. Najmaei, S.; Liu, Z.; Zhou, W.; Zou, X.; Shi, G.; Lei, S.; Yakobson, B. I.; Idrobo, J. C.; Ajayan, P. M.; Lou, J., Vapour phase growth and grain boundary structure of molybdenum disulphide atomic layers. Nat Mater 2013, 12 (8), 754-9.

23. Feng, J.; Qian, X. F.; Huang, C. W.; Li, J., Strain-engineered artificial atom as a broadspectrum solar energy funnel. Nat Photonics 2012, 6 (12), 865-871.

24. Py, M. A.; Haering, R. R., Structural destabilization induced by lithium intercalation in MoS2 and related compounds. Can J Phys 1983, 61 (1), 76-84. 
25. Lin, Y. C.; Dumcenco, D. O.; Huang, Y. S.; Suenaga, K., Atomic mechanism of the semiconducting-to-metallic phase transition in single-layered MoS2. Nat Nanotechnol 2014, 9 (5), 391-6.

26. Coehoorn, R.; Haas, C.; de Groot, R. A., Electronic structure of MoSe2, MoS2, and WSe2. II. The nature of the optical band gaps. Phys Rev B 1987, 35 (12), 6203-6206.

27. Chernikov, A.; Berkelbach, T. C.; Hill, H. M.; Rigosi, A.; Li, Y.; Aslan, O. B.; Reichman, D. R.; Hybertsen, M. S.; Heinz, T. F., Exciton Binding Energy and Nonhydrogenic Rydberg Series in Monolayer WS2. Phys Rev Lett 2014, 113 (7), 076802.

28. Beal, A. R.; Hughes, H. P., Kramers-Kronig analysis of the reflectivity spectra of $2 \mathrm{H}-$ MoS2 , 2H-MoSe2 and 2H-MoTe2. Journal of Physics C: Solid State Physics 1979, 12 (5), 881.

29. Bromley, R. A.; Murray, R. B.; Yoffe, A. D., The band structures of some transition metal dichalcogenides. III. Group VIA: trigonal prism materials. Journal of Physics C: Solid State Physics 1972, 5 (7), 759.

30. Carvalho, A.; Ribeiro, R. M.; Castro Neto, A. H., Band nesting and the optical response of two-dimensional semiconducting transition metal dichalcogenides. Phys Rev B 2013, 88 (11), 115205 .

31. Nair, R. R.; Blake, P.; Grigorenko, A. N.; Novoselov, K. S.; Booth, T. J.; Stauber, T.; Peres, N. M.; Geim, A. K., Fine structure constant defines visual transparency of graphene. Science 2008, 320 (5881), 1308.

32. Britnell, L.; Ribeiro, R. M.; Eckmann, A.; Jalil, R.; Belle, B. D.; Mishchenko, A.; Kim, Y.-J.; Gorbachev, R. V.; Georgiou, T.; Morozov, S. V.; Grigorenko, A. N.; Geim, A. K.; Casiraghi, C.; Neto, A. H. C.; Novoselov, K. S., Strong Light-Matter Interactions in Heterostructures of Atomically Thin Films. Science 2013, 340 (6138), 1311-1314.

33. Lee, C.; Yan, H.; Brus, L. E.; Heinz, T. F.; Hone, J.; Ryu, S., Anomalous Lattice Vibrations of Single- and Few-Layer MoS2. Acs Nano 2010, 4 (5), 2695-2700.

34. Zhao, W. J.; Ghorannevis, Z.; Kumar, A. K.; Pang, J. R.; Toh, M. L.; Zhang, X.; Kloc, C.; Tan, P. H.; Eda, G., Lattice dynamics in mono- and few-layer sheets of WS2 and WSe2 Nanoscale 2013, 5, 9677-9683.

35. Yoffe, A. D., Low-dimensional systems: Quantum size effects and electronic properties of semiconductor microcrystallites (zero-dimensional systems) and some quasi-two-dimensional systems. Adv Phys 2002, 51 (2), 799-890.

36. Cheiwchanchamnangij, T.; Lambrecht, W. R. L., Quasiparticle band structure calculation of monolayer, bilayer, and bulk MoS2. Phys Rev B 2012, 85 (20), 205302.

37. Tongay, S.; Zhou, J.; Ataca, C.; Liu, J.; Kang, J. S.; Matthews, T. S.; You, L.; Li, J.; Grossman, J. C.; Wu, J., Broad-range modulation of light emission in two-dimensional semiconductors by molecular physisorption gating. Nano Lett 2013, 13 (6), 2831-6.

38. Komsa, H. P.; Krasheninnikov, A. V., Effects of confinement and environment on the electronic structure and exciton binding energy of MoS2 from first principles. Phys Rev B 2012, 86 (24), 241201.

39. Yun, W. S.; Han, S. W.; Hong, S. C.; Kim, I. G.; Lee, J. D., Thickness and strain effects on electronic structures of transition metal dichalcogenides: 2H-MX2 semiconductors $(\mathrm{M}=\mathrm{Mo}$, $\mathrm{W} ; \mathrm{X}=\mathrm{S}, \mathrm{Se}, \mathrm{Te})$. Phys Rev B 2012, 85 (3), 033305.

40. Kuc, A.; Zibouche, N.; Heine, T., Influence of quantum confinement on the electronic structure of the transition metal sulfide TS2. Phys Rev B 2011, 83 (24), 245213.

41. Ramasubramaniam, A.; Naveh, D.; Towe, E., Tunable band gaps in bilayer transitionmetal dichalcogenides. Phys Rev B 2011, 84 (20), 205325. 
42. Jin, W.; Yeh, P.-C.; Zaki, N.; Zhang, D.; Sadowski, J. T.; Al-Mahboob, A.; van der Zande, A. M.; Chenet, D. A.; Dadap, J. I.; Herman, I. P.; Sutter, P.; Hone, J.; Osgood, R. M., Jr., Direct Measurement of the Thickness-Dependent Electronic Band Structure of MoS2 Using Angle-Resolved Photoemission Spectroscopy. Phys Rev Lett 2013, 111 (10), 106801.

43. Klein, A.; Tiefenbacher, S.; Eyert, V.; Pettenkofer, C.; Jaegermann, W., Electronic band structure of single-crystal and single-layer WS2: Influence of interlayer van der Waals interactions. Phys Rev B 2001, 64 (20), 205416.

44. Zhao, W.; Ribeiro, R. M.; Toh, M.; Carvalho, A.; Kloc, C.; Castro Neto, A. H.; Eda, G., Origin of indirect optical transitions in few-layer MoS2, WS2, and WSe2. Nano Lett 2013, 13 (11), 5627-34.

45. Peelaers, H.; Van de Walle, C. G., Effects of strain on band structure and effective masses in MoS2. Phys Rev B 2012, 86 (24), 241401(R).

46. EL-MAHALAWY, S. H.; EVANS, B. L., The Thermal Expansion of 2H-MoS2, 2HMoSe 2 and $2 \mathrm{H}-\mathrm{WSe} 2$ between 20 and $800^{\circ} \mathrm{C}$. J. Appl. Cryst. 1976, 9, 403.

47. MURRAY, R.; EVANS, B. L., The Thermal Expansion of 2H-MoS2 and 2H-WSe2 between 10 and $320 \mathrm{~K}$. J. Appl. Cryst. 1979, 12, 312.

48. Sahin, H.; Tongay, S.; Horzum, S.; Fan, W.; Zhou, J.; Li, J.; Wu, J.; Peeters, F. M., Anomalous Raman spectra and thickness-dependent electronic properties of WSe2. Phys Rev B 2013, 87 (16), 165409.

49. Eda, G.; Chhowalla, M., Chemically derived graphene oxide: towards large-area thinfilm electronics and optoelectronics. Adv Mater 2010, 22 (22), 2392-415.

50. Coleman, J. N.; Lotya, M.; O'Neill, A.; Bergin, S. D.; King, P. J.; Khan, U.; Young, K.; Gaucher, A.; De, S.; Smith, R. J.; Shvets, I. V.; Arora, S. K.; Stanton, G.; Kim, H. Y.; Lee, K.; Kim, G. T.; Duesberg, G. S.; Hallam, T.; Boland, J. J.; Wang, J. J.; Donegan, J. F.; Grunlan, J. C.; Moriarty, G.; Shmeliov, A.; Nicholls, R. J.; Perkins, J. M.; Grieveson, E. M.; Theuwissen, K.; McComb, D. W.; Nellist, P. D.; Nicolosi, V., Two-dimensional nanosheets produced by liquid exfoliation of layered materials. Science 2011, 331 (6017), 568-71.

51. Nicolosi, V.; Chhowalla, M.; Kanatzidis, M. G.; Strano, M. S.; Coleman, J. N., Liquid Exfoliation of Layered Materials. Science 2013, 340 (6139).

52. King, L. A.; Zhao, W.; Chhowalla, M.; Riley, D. J.; Eda, G., Photoelectrochemical properties of chemically exfoliated MoS2. Journal of Materials Chemistry A 2013, 1 (31), 89358941.

53. Joensen, P.; Crozier, E. D.; Alberding, N.; Frindt, R. F., A study of single-layer and restacked MoS2 by X-ray diffraction and X-ray absorption spectroscopy. Journal of Physics C: Solid State Physics 1987, 20 (26), 4043.

54. Eda, G.; Yamaguchi, H.; Voiry, D.; Fujita, T.; Chen, M.; Chhowalla, M., Photoluminescence from chemically exfoliated MoS2. Nano Lett 2011, 11 (12), 5111-6.

55. Heising, J.; Kanatzidis, M. G., Structure of Restacked MoS2 and WS2 Elucidated by Electron Crystallography. Journal of the American Chemical Society 1999, 121 (4), 638-643.

56. Eda, G.; Fujita, T.; Yamaguchi, H.; Voiry, D.; Chen, M.; Chhowalla, M., Coherent atomic and electronic heterostructures of single-layer MoS2. Acs Nano 2012, 6 (8), 7311-7.

57. Voiry, D.; Yamaguchi, H.; Li, J.; Silva, R.; Alves, D. C. B.; Fujita, T.; Chen, M.; Asefa, T.; Shenoy, V. B.; Eda, G.; Chhowalla, M., Enhanced catalytic activity in strained chemically exfoliated WS2 nanosheets for hydrogen evolution. Nat Mater 2013, 12 (9), 850-855. 
58. Kan, M.; Wang, J. Y.; Li, X. W.; Zhang, S. H.; Li, Y. W.; Kawazoe, Y.; Sun, Q.; Jena, P., Structures and Phase Transition of a MoS2 Monolayer. The Journal of Physical Chemistry C 2014, 118 (3), 1515-1522.

59. Evans, B. L.; Thompson, K. T., The photovoltage in thin crystals of MoS2. Journal of Physics D: Applied Physics 1968, I (12), 1619.

60. Kozawa, D.; Kumar, R.; Carvalho, A.; Kumar Amara, K.; Zhao, W.; Wang, S.; Toh, M.; Ribeiro, R. M.; Castro Neto, A. H.; Matsuda, K.; Eda, G., Photocarrier relaxation pathway in two-dimensional semiconducting transition metal dichalcogenides. Nat Commun 2014, 5, 4543.

61. Qiu, D. Y.; da Jornada, F. H.; Louie, S. G., Optical spectrum of MoS2: many-body effects and diversity of exciton states. Phys Rev Lett 2013, 111 (21), 216805.

62. Kośmider, K.; Fernández-Rossier, J., Electronic properties of the MoS2-WS2 heterojunction. Phys Rev B 2013, 87 (7), 075451.

63. Terrones, H.; Lopez-Urias, F.; Terrones, M., Novel hetero-layered materials with tunable direct band gaps by sandwiching different metal disulfides and diselenides. Sci Rep 2013, 3, 1549 . 

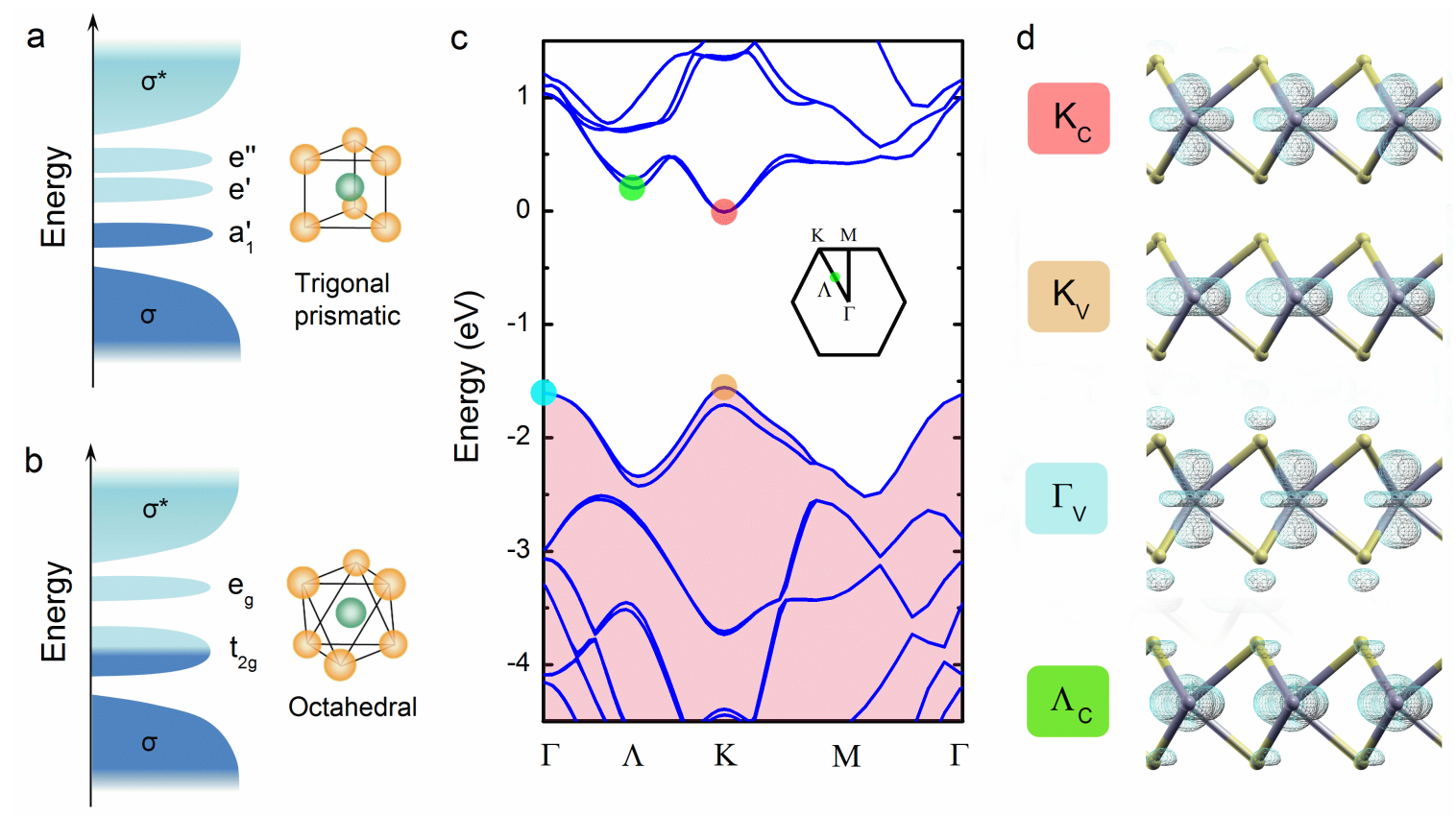

Figure 1. Schematic illustration of orbital filling of group 6 TMDs with trigonal prismatic (a) and octahedral (b) coordination. The filled and unfilled orbitals are shaded with dark and light blue, respectively. In trigonal prismatic $(2 \mathrm{H})$ coordination, the $\mathrm{d}$ orbitals split into three groups, namely $d_{z}^{2}\left(a_{1}\right), d_{x-y, x y}^{2}{ }^{2}\left(e^{\prime}\right)$ and $d_{x z, y z}\left(e^{\prime \prime}\right)$. These orbitals are located within the bonding $(\sigma)$ and antibonding $\left(\sigma^{*}\right)$ states. The energy gap between the fully filled and unfilled orbitals makes it a semiconductor. In octahedral (1T) coordination, the d orbitals split into $d_{x y, y z, z x}\left(t_{2 g}\right)$ and $d_{z}^{2}{ }_{3 x-y}^{2}$ $\left(\mathrm{e}_{\mathrm{g}}\right)$ bands. This phase is metallic because the $\mathrm{t}_{2 \mathrm{~g}}$ orbital is half-filled. (c) Calculated electronic band structure of monolayer $\mathrm{MoS}_{2}$. The first Brillouin zone is shown in the inset. (d) Electron distribution for states at the four extrema in the band structure indicated by colored dots in (c). It can be seen that the $d$ orbital contribution is dominant at the $\mathrm{K}_{\mathrm{c}} / \mathrm{K}_{\mathrm{v}}$ point while contributions from the $\mathrm{p}$ orbitals from chalcogen is evident at the $\Gamma_{\mathrm{v}}$ and $\Lambda_{\mathrm{c}}$ point. 

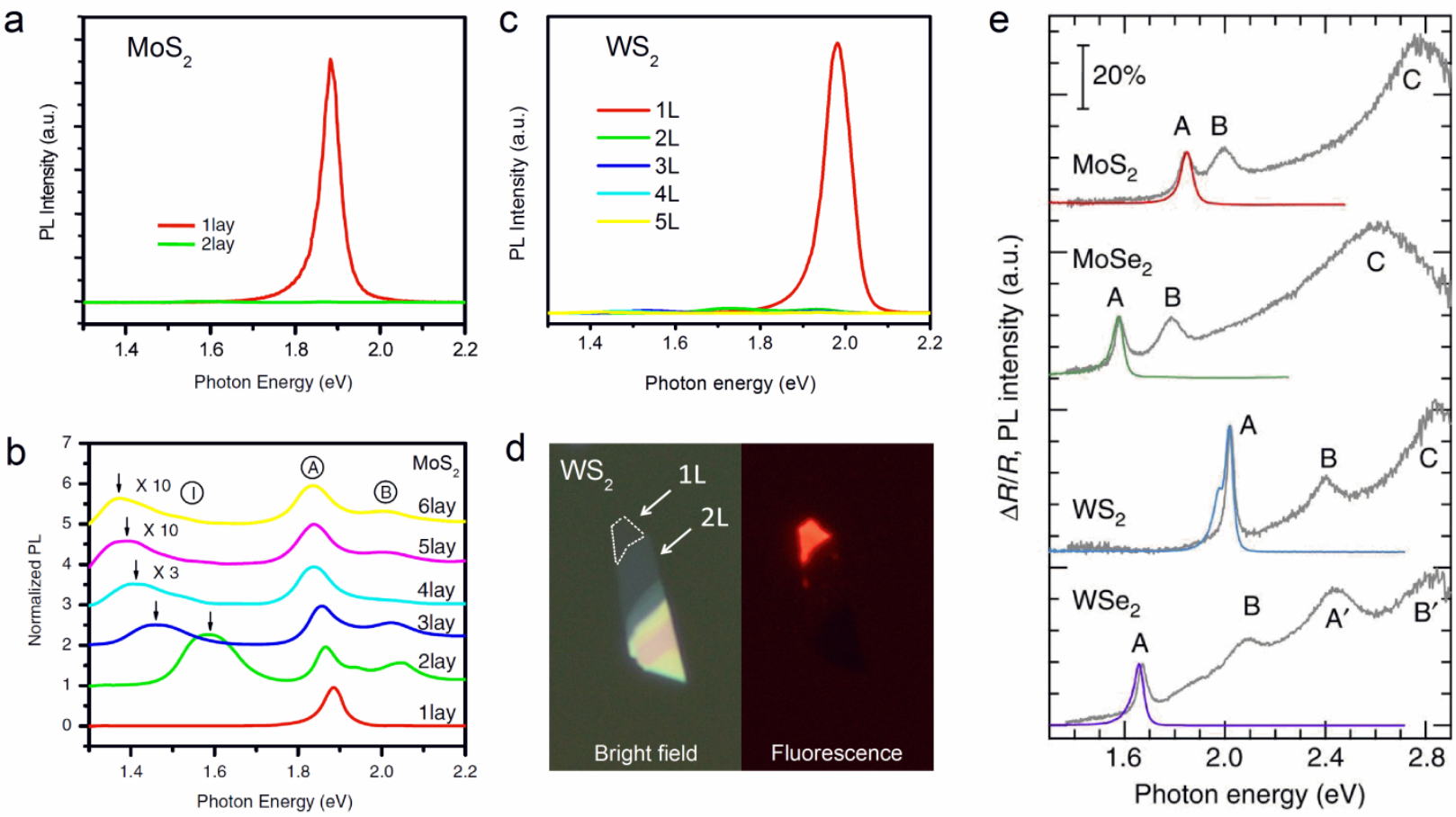

Figure 2 (a) PL spectra of mechanically exfoliated monolayer and bilayer $2 \mathrm{H}-\mathrm{MoS}_{2}$. Monolayer $\mathrm{MoS}_{2}$ exhibits significantly brighter PL compared to bilayer $\mathrm{MoS}_{2}$. (b) Normalized PL spectra of 1-6 $\mathrm{L} \mathrm{MoS}_{2}$. The direct gap PL, marked with A, is not sensitive to the layer number. In contrast, indirect gap emission peak, marked with I, decreases in energy with increasing layer number. (Adapted with permission from ref 10. Copyright 2010 American Physical Society). (c) PL spectra of 1-5 $\mathrm{L} \mathrm{WS}_{2}$. (d) Bright field optical and fluorescence images of mechanically exfoliated $\mathrm{WS}_{2}$. The monolayer region shows bright fluorescence compared to other multilayer regions. (e) Normalized PL spectra (colored plots) and differential reflectance spectra (grey curves) of monolayer $\mathrm{MX}_{2}$ flakes on a quartz substrate. The scale bar indicates $20 \%$ absorption based on the differential reflectance spectra. (Adapted with permission from ref 60. Macmillan Publishers Ltd. Copyright 2014.) 

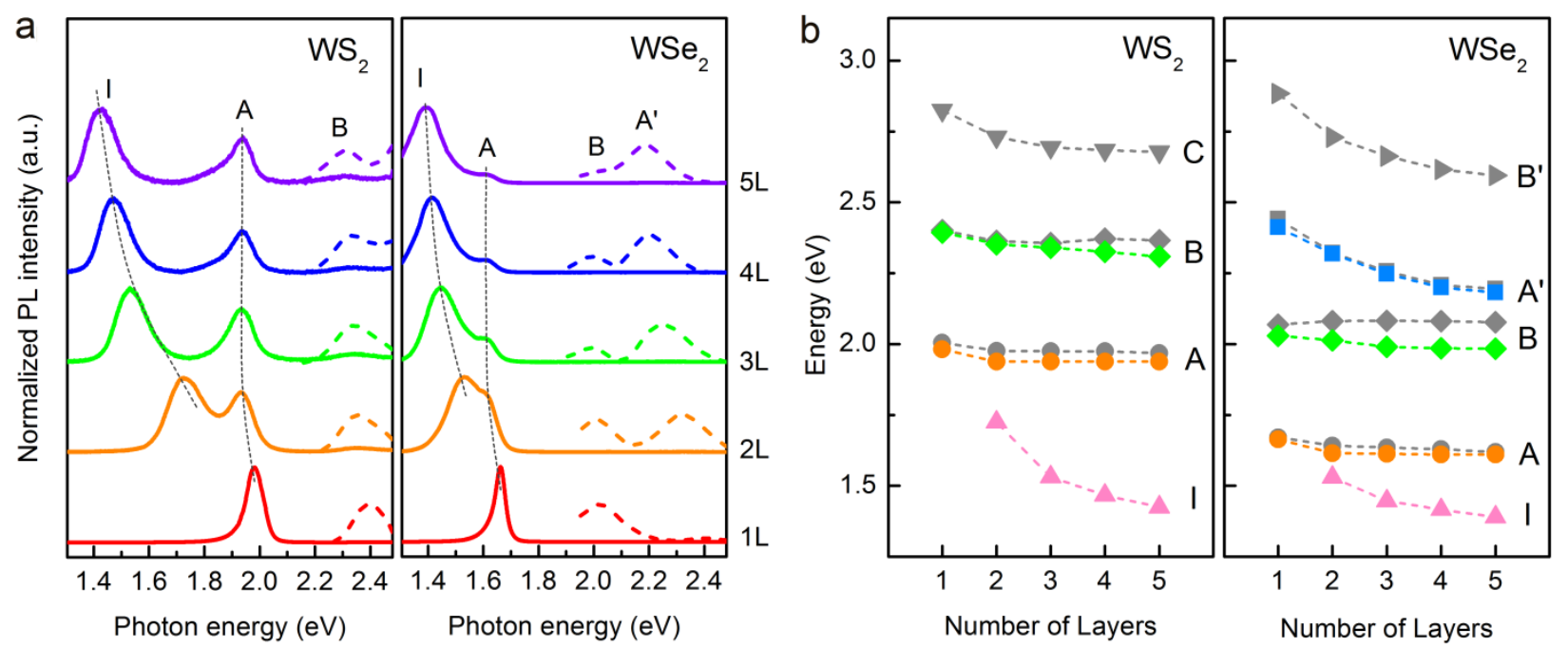

Figure 3. (a) Normalized PL spectra of 1-5 $\mathrm{L} \mathrm{WS}_{2}$ (left) and $\mathrm{WSe}_{2}$ (right), respectively. The shift of the PL peaks corresponding to the $\operatorname{direct}(\mathrm{A}, \mathrm{B})$ and indirect transitions (I) is similar to that of $\mathrm{MoS}_{2}$ shown in Figure 2b. (b) PL (color) and absorption (gray) peak energies of $\mathrm{WS}_{2}$ (left) and $\mathrm{WSe}_{2}$ (right) as a function of the number of layers. The letters I, A, B, A', B', and C refer to the peaks as labeled in Figures 2e and 3a. (Adapted with permission from ref 12. Copyright 2012 American Chemical Society) 
a
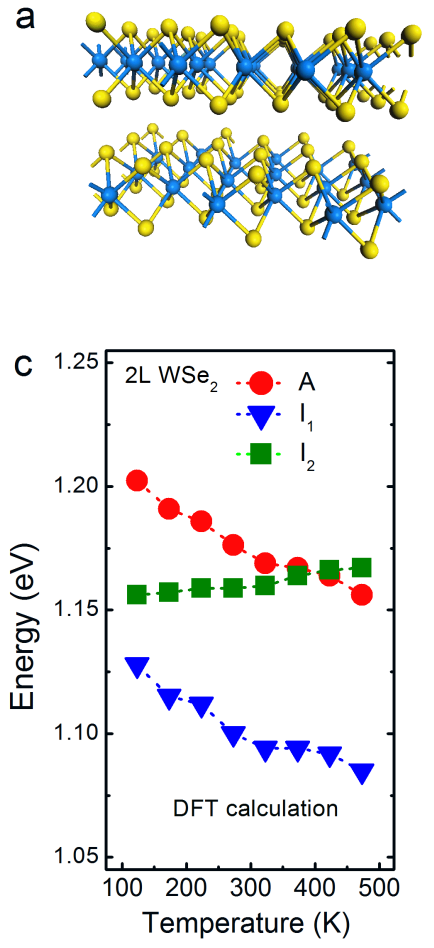

b

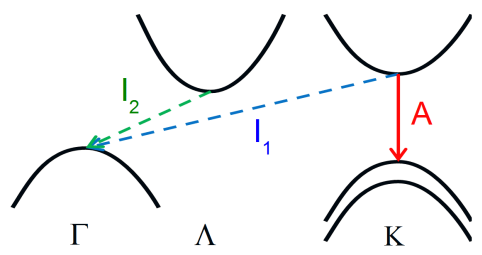

d
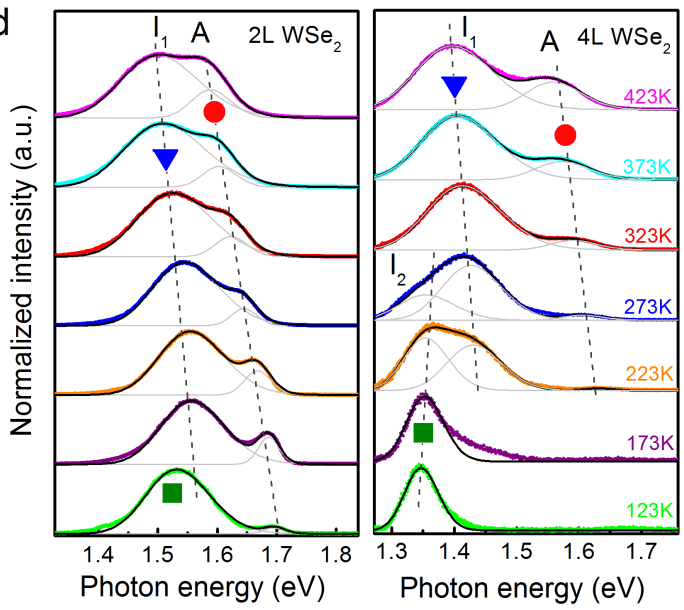

Figure 4. (a) Structure of bilayer $2 \mathrm{H}-\mathrm{MX}_{2}$. (b) Schematic illustration of the electronic band structure of bilayer $2 \mathrm{H}-\mathrm{MX}_{2}$. The red arrow labeled A represents the optical transition of direct band gap at $\mathrm{K}$ point. The dashed arrow labeled $\mathrm{I}_{1}$ and $\mathrm{I}_{2}$ indicate the possible indirect emissions. (c) Calculated direct and indirect transition energies as a function of temperature for bilayer $\mathrm{WSe}_{2}$. (d) Normalized PL spectra of bilayer (left) and tetralayer (right) $\mathrm{WSe}_{2}$ measured at different temperatures. The colored plots are the experimental data. The temperature-dependent shift of the direct (A: red circle) and indirect emission peaks $\left(\mathrm{I}_{1}\right.$ : blue triangle; $\mathrm{I}_{2}$ : green squre) are indicated with dashed lines. (c,d: Adapted with permission from ref 44. Copyright 2013 American Chemical Society) 
a

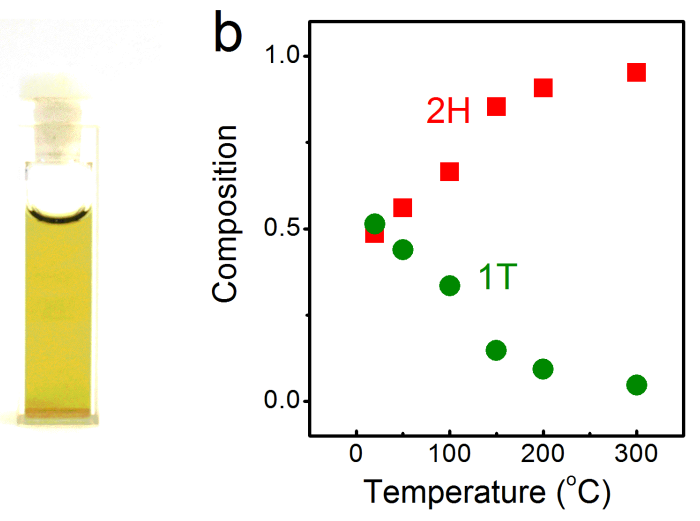

d
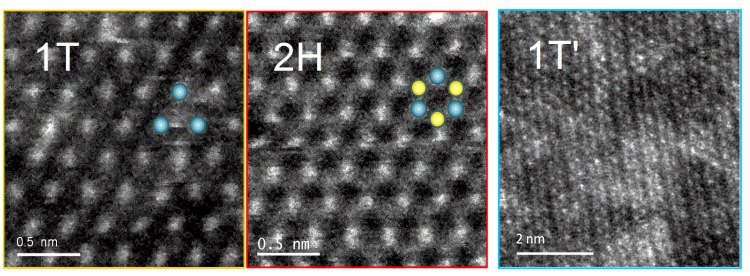

e

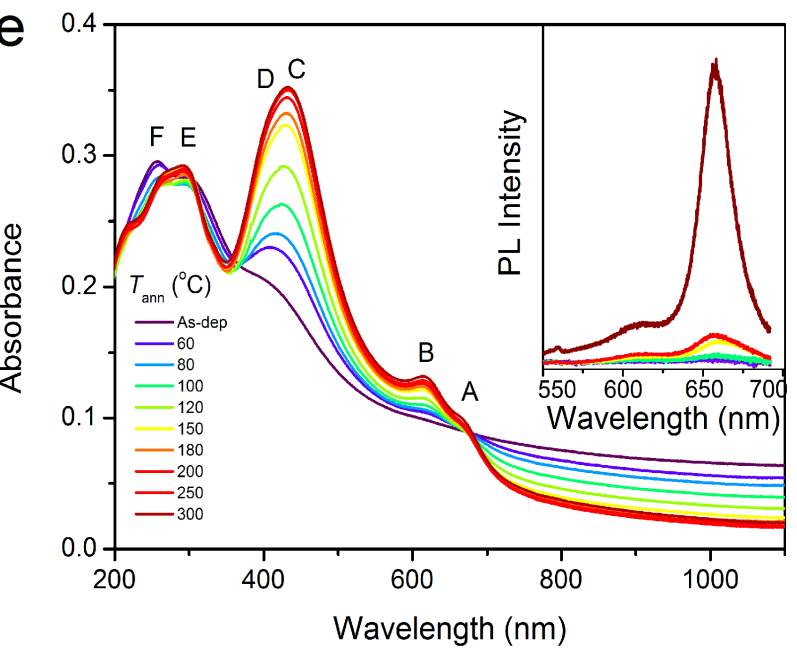

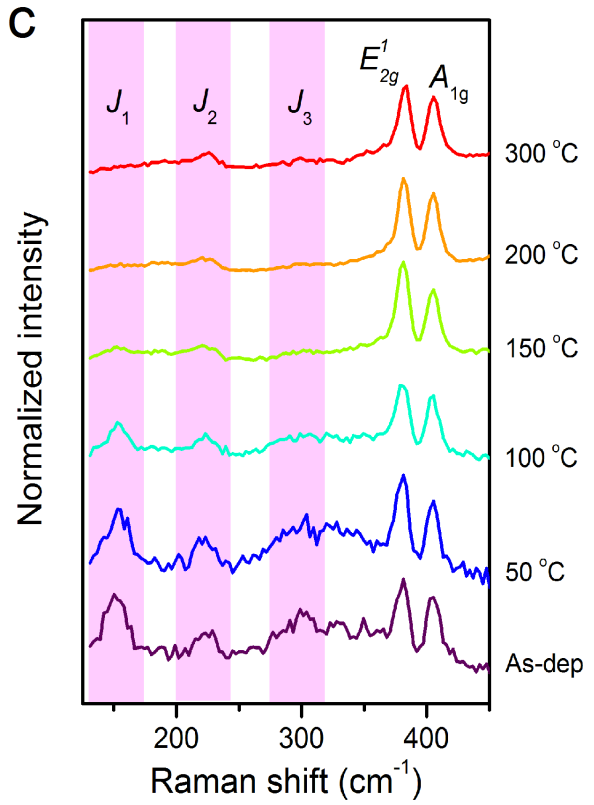

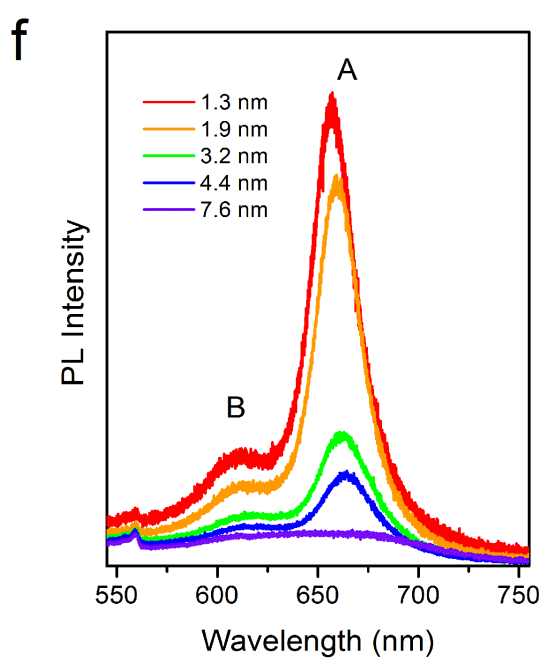

Figure 5. (a) Photograph showing an aqueous suspension of chemically exfoliated $\mathrm{MoS}_{2}$. (b) Relative composition of $2 \mathrm{H}$ and $1 \mathrm{~T}$ components as a function of annealing temperature extracted from XPS spectra. (c) Raman spectra of chemically exfoliated $\mathrm{MoS}_{2}$ thin films with a thickness of $\sim 4 \mathrm{~nm}$ annealed at various temperatures. The characteristic Raman peaks for 1T/1T' phase $\left(\mathrm{J}_{1}, \mathrm{~J}_{2}\right.$, and $\left.\mathrm{J}_{3}\right)$ are indicated in the shaded regions. (d) High resolution STEM images of $2 \mathrm{H}, 1 \mathrm{~T}$, and $1 \mathrm{~T}^{\prime}$ phases. The blue and yellow balls indicate the position of Mo and $\mathrm{S}$ atoms. Adapted 
with permission from ref 56. Copyright 2012 American Chemical Society. (e) Absorption and PL (insert) spectra of $\mathrm{MoS}_{2}$ thin films with a thickness of $\sim 1.3 \mathrm{~nm}$ annealed at various temperatures. The evolution of the spectra with increasing temperature indicates the restoration of the $2 \mathrm{H}$ phase from the $1 \mathrm{~T}$ phase. (f) PL spectra of $\mathrm{MoS}_{2}$ thin films with average thicknesses ranging from 1.3 to $7.6 \mathrm{~nm}$. (a,b,e,f: Adapted with permission from ref 54. Copyright 2011 American Chemical Society) 


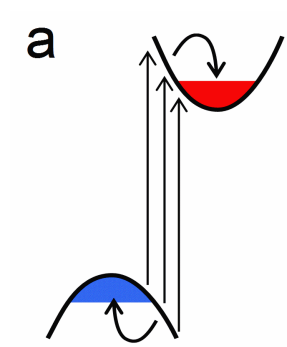

Г

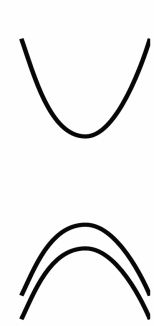

$\mathrm{K}$
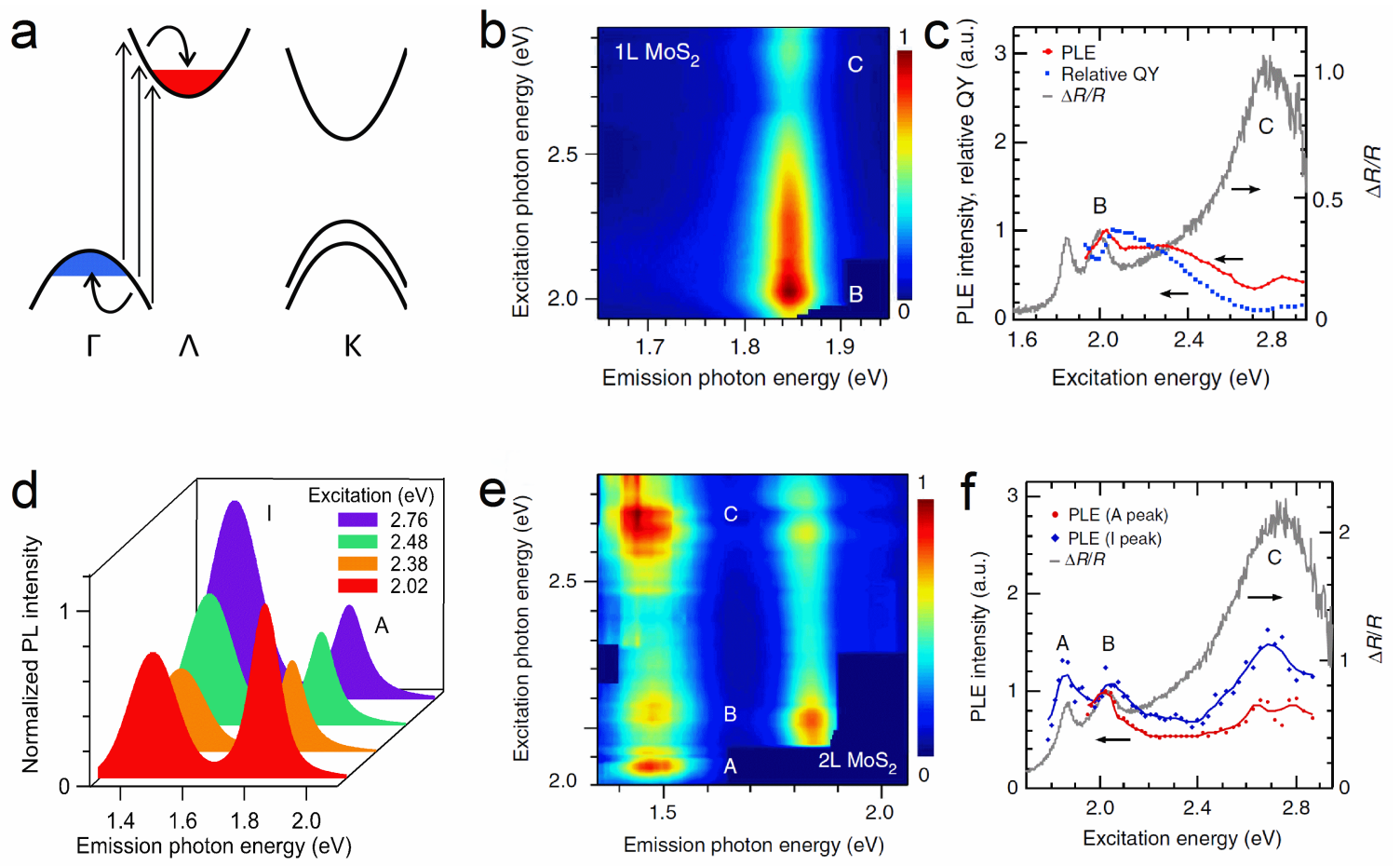

Figure 6. (a) Schematic showing the band structure of monolayer $\mathrm{MoS}_{2}$. At the band nesting region, the photoexcited electron-hole pairs spontaneously form indirect excitons with electrons and holes relaxing with opposite momentum to the nearest band extrema. PLE intensity map (b) and spectrum (c) for band gap emission for monolayer $\mathrm{MoS}_{2}$. Differential reflectance spectrum is shown for comparison in gray plot. The PLE spectrum is normalized to the B exciton peak of monolayer $\mathrm{MoS}_{2}$. (d) PL spectra of $\mathrm{MoS}_{2}$ excited at different excitation energies. PLE intensity map (e) and spectrum (f) for bilayer $\mathrm{MoS}_{2}$. The differential reflectance spectrum is shown for comparison in gray plot. The PLE spectrum of the A peak is normalized to the B exciton peak of the differential reflectance. The PLE spectrum of the I peak is multiplied by the same factor as the PLE spectrum of the A peak. (b,c,e,f: Adapted with permission from ref 60. Macmillan Publishers Ltd. Copyright 2014.) 